Acta Crystallographica Section E

Structure Reports

Online

ISSN 1600-5368

\section{(E)-1-(2-Furyl)-3-(2,4,6-trimethoxy- phenyl)prop-2-en-1-one}

\section{Hoong-Kun Fun, ${ }^{a} * \ddagger$ Thitipone Suwunwong, ${ }^{\mathrm{b}}$ Suchada Chantrapromma $^{\mathrm{b}} \S$ and Chatchanok Karalai ${ }^{\mathrm{b}}$}

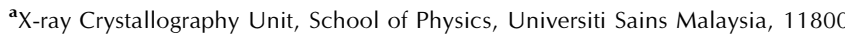
USM, Penang, Malaysia, and ${ }^{\mathbf{b}}$ Crystal Materials Research Unit, Department of Chemistry, Faculty of Science, Prince of Songkla University, Hat-Yai, Songkhla 90112, Thailand

Correspondence e-mail: hkfun@usm.my

Received 31 August 2010; accepted 6 September 2010

Key indicators: single-crystal X-ray study; $T=100 \mathrm{~K}$; mean $\sigma(\mathrm{C}-\mathrm{C})=0.002 \AA$; $R$ factor $=0.041 ; w R$ factor $=0.113 ;$ data-to-parameter ratio $=15.5$.

In the title heteroaryl chalcone derivative, $\mathrm{C}_{16} \mathrm{H}_{16} \mathrm{O}_{5}$, the dihedral angle between the furan and benzene rings is $14.45(6)^{\circ}$. The three methoxy groups are almost coplanar with their attached benzene ring $[\mathrm{C}-\mathrm{C}-\mathrm{O}-\mathrm{C}$ torsion angles $=2.07(17),-5.04(17)$ and $\left.2.85(16)^{\circ}\right]$. An intramolecular $\mathrm{C}-$ $\mathrm{H} \cdot \mathrm{OO}$ hydrogen bond occurs. In the crystal, adjacent molecules are linked into $\mathrm{X}$-shaped chains along the $c$ axis by weak $\mathrm{C}-\mathrm{H} \cdots \mathrm{O}$ (enone) interactions. These chains are stacked along the $b$ axis. C. . O [3.3308 (13)-3.4123 (14) A] short contacts are also observed.

\section{Related literature}

For bond-length data, see: Allen et al. (1987). For hydrogenbond motifs, see: Bernstein et al. (1995). For related structures, see: Chantrapromma et al. (2009); Suwunwong et al. (2009. For background to and applications of chalcones and heteroaryl chalcones, see: Gaber et al. (2008); Go et al. (2005); Jung et al. (2008); Ng et al. (2009); Ni et al. (2004); Nowakowska (2007); Patil \& Dharmaprakash (2008) and Tewtrakul et al. (2003). For the stability of the temperature controller used in the data collection, see Cosier \& Glazer, (1986).<smiles>COc1cc(OC)c(/C=C/C(=O)c2ccco2)c(OC)c1</smiles>

† Thomson Reuters ResearcherID: A-3561-2009.

§ Additional correspondence author, e-mail: suchada.c@psu.ac.th. Thomson Reuters ResearcherID: A-5085-2009.

\section{Experimental}

\begin{tabular}{|c|c|}
\hline & $V=269068$ \\
\hline $\begin{array}{l}C_{16} \boldsymbol{H}_{16} \mathrm{U}_{5} \\
M_{r}=288.29\end{array}$ & $\begin{array}{l}V=2090.00(0) A \\
Z=8\end{array}$ \\
\hline Monoclinic, $C 2 / c$ & Mo $K \alpha$ radiation \\
\hline$a=38.5688(5) \AA$ & $\mu=0.11 \mathrm{~mm}^{-1}$ \\
\hline$b=3.93493(5) \AA$ & $T=100 \mathrm{~K}$ \\
\hline$c=18.2638(3) \AA$ & $0.41 \times 0.15 \times 0.09 \mathrm{~mm}$ \\
\hline
\end{tabular}

\section{Data collection \\ Bruker APEXII CCD \\ diffractometer \\ Absorption correction: multi-scan (SADABS; Bruker, 2005)

\begin{abstract}
20657 measured reflections 3941 independent reflections 3077 reflections with $I>2 \sigma(I)$ $R_{\text {int }}=0.038$
\end{abstract}

$T_{\min }=0.957, T_{\max }=0.990$

Refinement

$R\left[F^{2}>2 \sigma\left(F^{2}\right)\right]=0.041$

$w R\left(F^{2}\right)=0.113$

$S=1.06$

3941 reflections

254 parameters

All $\mathrm{H}$-atom parameters refined

$\Delta \rho_{\max }=0.35{\mathrm{e} \AA^{-3}}^{-3}$

$\Delta \rho_{\min }=-0.24$ e $\AA^{-3}$

Table 1

Hydrogen-bond geometry $\left(\AA{ }^{\circ}\right)$.

\begin{tabular}{lllll}
\hline$D-\mathrm{H} \cdots A$ & $D-\mathrm{H}$ & $\mathrm{H} \cdots A$ & $D \cdots A$ & $D-\mathrm{H} \cdots A$ \\
\hline $\mathrm{C} 1-\mathrm{H} 1 \cdots \mathrm{O} 2{ }^{\mathrm{i}}$ & $0.956(15)$ & $2.496(15)$ & $3.3512(14)$ & $148.9(13)$ \\
$\mathrm{C} 6-\mathrm{H} 6 \cdots \mathrm{O} 5$ & $0.965(15)$ & $2.260(14)$ & $2.8197(15)$ & $116.0(11)$ \\
$\mathrm{C} 14-\mathrm{H} 14 A \cdots \mathrm{O} 4{ }^{\mathrm{ii}}$ & $0.975(15)$ & $2.589(16)$ & $3.4462(14)$ & $146.7(11)$ \\
$\mathrm{C} 15-\mathrm{H} 15 A \cdots \mathrm{O} 1^{\mathrm{iii}}$ & $0.989(16)$ & $2.546(16)$ & $3.4293(18)$ & $148.6(12)$ \\
$\mathrm{C} 16-\mathrm{H} 16 A \cdots \mathrm{O} 1^{\mathrm{iii}}$ & $0.982(16)$ & $2.575(16)$ & $3.4120(16)$ & $143.0(12)$ \\
\hline Symmetry codes: $(\mathrm{i})-x+\frac{1}{2},-y+\frac{1}{2},-z+2 ;(\mathrm{ii})-x,-y,-z+1 ;($ iii $) x,-y, z-\frac{1}{2}$.
\end{tabular}

Data collection: APEX2 (Bruker, 2005); cell refinement: SAINT (Bruker, 2005); data reduction: $S A I N T$; $\operatorname{program}(\mathrm{s})$ used to solve structure: SHELXTL (Sheldrick, 2008); program(s) used to refine structure: SHELXTL; molecular graphics: SHELXTL; software used to prepare material for publication: SHELXTL and PLATON (Spek, 2009).

The authors thank the Thailand Research Fund (TRF) for a research grant (RSA 5280033) and the Prince of Songkla University for financial support. The authors also thank Universiti Sains Malaysia for the Research University Golden Goose grant No. 1001/PFIZIK/811160. Mr Teerasak Anantapong, Department of Biotechnology, Faculty of Agro-Industry, Prince of Songkla University, is acknowledged for the bacterial assay.

Supplementary data and figures for this paper are available from the IUCr electronic archives (Reference: HB5630).

\title{
References
}

Allen, F. H., Kennard, O., Watson, D. G., Brammer, L., Orpen, A. G. \& Taylor, R. (1987). J. Chem. Soc. Perkin Trans. 2, pp. S1-19.

Bernstein, J., Davis, R. E., Shimoni, L. \& Chang, N.-L. (1995). Angew. Chem. Int. Ed. Engl. 34, 1555-1573.

Bruker (2005). APEX2, SAINT and SADABS. Bruker AXS Inc., Madison, Wisconsin, USA.

Chantrapromma, S., Suwunwong, T., Karalai, C. \& Fun, H.-K. (2009). Acta Cryst. E65, o893-0894.

Cosier, J. \& Glazer, A. M. (1986). J. Appl. Cryst. 19, 105-107. 


\section{organic compounds}

Gaber, M., El-Daly, S. A., Fayed, T. A. \& El-Sayed, Y. S. (2008). Opt. Laser Technol. 40, 528-537.

Go, M.-L., Wu, X. \& Liu, X.-L. (2005). Curr. Med. Chem. 12, 483-499.

Jung, Y. J., Son, K. I., Oh, Y. E. \& Noh, D. Y. (2008). Polyhedron. 27, 861-867.

Ng, L.-T., Ko, H.-H. \& Lu, T.-M. (2009). Bioorg. Med. Chem. 17, 4360-4366.

Ni, L., Meng, C. Q. \& Sikorski, J. A. (2004). Exp. Opin. Ther. Patents, 14, 16691691.

Nowakowska, Z. (2007). Eur. J. Med. Chem. 42, 125-137.
Patil, P. S. \& Dharmaprakash, S. M. (2008). Mater. Lett. 62, 451-453.

Sheldrick, G. M. (2008). Acta Cryst. A64, 112-122.

Spek, A. L. (2009). Acta Cryst. D65, 148-155.

Suwunwong, T., Chantrapromma, S., Pakdeevanich, P. \& Fun, H.-K. (2009). Acta Cryst. E65, o1575-o1576.

Tewtrakul, S., Subhadhirasakul, S., Puripattanavong, J. \& Panphadung, T. (2003). Songklanakarin J. Sci. Technol. 25, 503-508. 


\section{supporting information}

Acta Cryst. (2010). E66, o2559-o2560］[oi:10.1107/S1600536810035762]

\section{(E)-1-(2-Furyl)-3-(2,4,6-trimethoxyphenyl)prop-2-en-1-one}

\section{Hoong-Kun Fun, Thitipone Suwunwong, Suchada Chantrapromma and Chatchanok Karalai}

\section{S1. Comment}

Chalcone and heteroaryl chalcones are very interesting due to their variety of applications with biological activities. Many of them possess analgesic, anti-inflammatory and antibacterial properties (Go et al., 2005; Ni et al., 2004; Nowakowska, 2007) as well as HIV-1 protease inhibitory (Tewtrakul et al., 2003) and tyrosinase inhibitory (Ng et al., 2009) activities. Moreover synthetic chalcones and heteroaryl chalcones have also been found to exhibit non-linear optical (Patil \& Dharmaprakash, 2008), fluorescent (Jung et al., 2008) and laser properties (Gaber et al., 2008) . In continuing our on-going research on antibacterial activities and fluorescence properties of chalcones and heteroaryl chalcone derivatives, the title heteroaryl chalcone was synthesized in order to study its antibacterial and fluorescence properties. However our results show that (I) do not possess fluorescence property. In addition our biological testing found that (I) was inactive against the tested bacteria strains which are Bacillus subtilis, Enterococcus faecalis, Staphylococcus aureus, Methicillin-Resistant Staphylococcus aureus, Vancomycin-Resistant Enterococcus faecalis, Pseudomonas aeruginosa, Salmonella typhi and Shigella sonnei. Herein we report the crystal structure of (I).

The molecule of the title heteroaryl chalcone (Fig. 1) exists in an $E$ configuration with respect to the $\mathrm{C} 6=\mathrm{C} 7$ double bond [1.3512 (16) $\AA]$ with the $\mathrm{C} 5-\mathrm{C} 6-\mathrm{C} 7-\mathrm{C} 8$ torsion angle being $-176.44(12)^{\circ}$. The whole molecule is slightly twisted with the dihedral angle between the furan and benzene rings being $14.45(6)^{\circ}$. Atoms of the propenone unit (C5, C6, C7 and O1) lie on the same plane [r.m.s. 0.0179 (1)]. This plane makes dihedral angles of $11.38(8)$ and $9.12(8)^{\circ}$ with furan and phenyl rings, respectively. All the three substituted methoxy groups of 2,4,6-trimethoxyphenyl unit are almost coplanar with the phenyl ring as indicated by torsion angles $\mathrm{C} 14-\mathrm{O} 3-\mathrm{C} 9-\mathrm{C} 10=2.07(17)^{\circ}, \mathrm{C} 15-\mathrm{O} 4-\mathrm{C} 11-\mathrm{C} 12=$ $-5.04(17)^{\circ}$ and $\mathrm{C} 16-\mathrm{O} 5-\mathrm{C} 13-\mathrm{C} 12=2.85(16)^{\circ}$. In the structure, a weak intramolecular $\mathrm{C} 6-\mathrm{H} 6 \cdots \mathrm{O} 5$ interaction generates an S(6) ring motif (Bernstein et al., 1995) (Table 1). The bond lengths have normal values (Allen et al., 1987) and bond lengths and angles are comparable with its related structures (Chantrapromma et al., 2009; Suwunwong et al., 2009).

In the crystal packing, all the three methoxy groups involve in weak intermolecular $\mathrm{C}-\mathrm{H} \cdots \mathrm{O}$ interactions (Table 1). The adjacent molecules are linked into $\mathrm{X}$-shape chains along the $c$ axis through the enone unit by weak $\mathrm{C}-\mathrm{H} \cdots \mathrm{O}$ interactions (Fig. 2, Table 1). The adjacent chains are arranged into face-to-face manner (Fig. 3) and stacked along the $b$ axis (Fig. 3). The crystal is further stabilized by $\mathrm{C} \cdots \mathrm{O}[3.3308$ (13)-3.4123 (14) $\AA]$ short contacts.

\section{S2. Experimental}

The title compound was prepared by the condensation of the solution of 2-furyl methylketone ( $2 \mathrm{mmol}, 0.22 \mathrm{~g}$ ) in ethanol $(15 \mathrm{ml})$ and 2,4,6-trimethoxybenzaldehyde $(2 \mathrm{mmol}, 0.40 \mathrm{~g})$ in ethanol $(15 \mathrm{ml})$ in the presence of $20 \% \mathrm{NaOH}(\mathrm{aq}) 5 \mathrm{ml}$ at $278 \mathrm{~K}$ for $5 \mathrm{hr}$. The resulting solid which was obtained was further collected by filtration, washed with distilled water and dried in air. Colorless blocks of (I) were recrystalized from acetone/ethanol $(1: 1 v / v)$ by the slow evaporation of the solvent at room temperature after several days, Mp. 390-391 K. 


\section{supporting information}

\section{S3. Refinement}

All $\mathrm{H}$ atoms were located in difference maps and refined isotropically. The highest residual electron density peak is located at $0.63 \AA$ from $\mathrm{C} 10$ and the deepest hole is located at $1.12 \AA$ from $\mathrm{C} 2$.

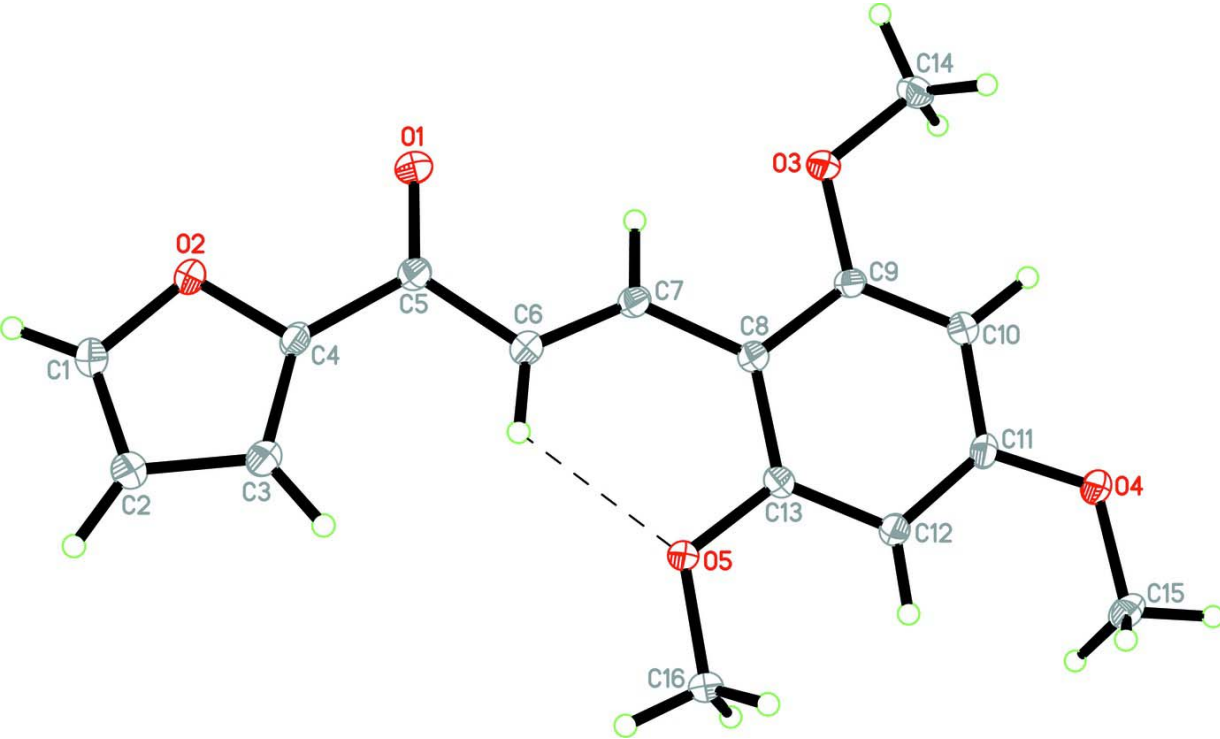

\section{Figure 1}

The molecular structure of (I), showing 50\% probability displacement ellipsoids. Weak intramolecular interactions are shown as dashed lines. 


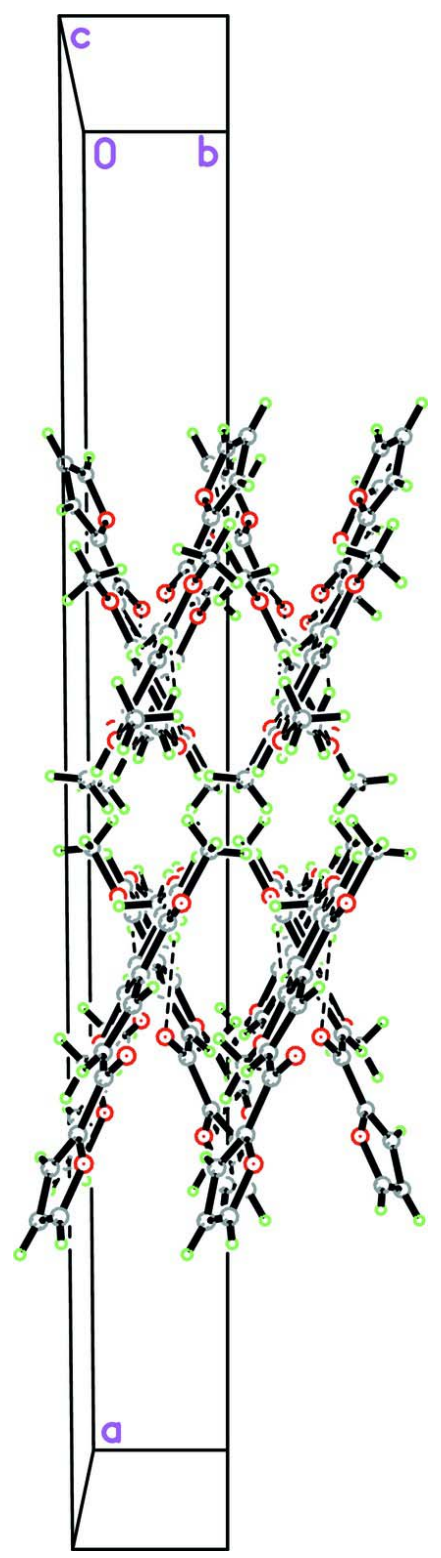

Figure 2

The crystal packing of (I) viewed along the $c$ axis, showing X-chains running along the $c$ axis. Weak $\mathrm{C}-\mathrm{H} \cdots \mathrm{O}$ interactions are shown as dashed lines. 


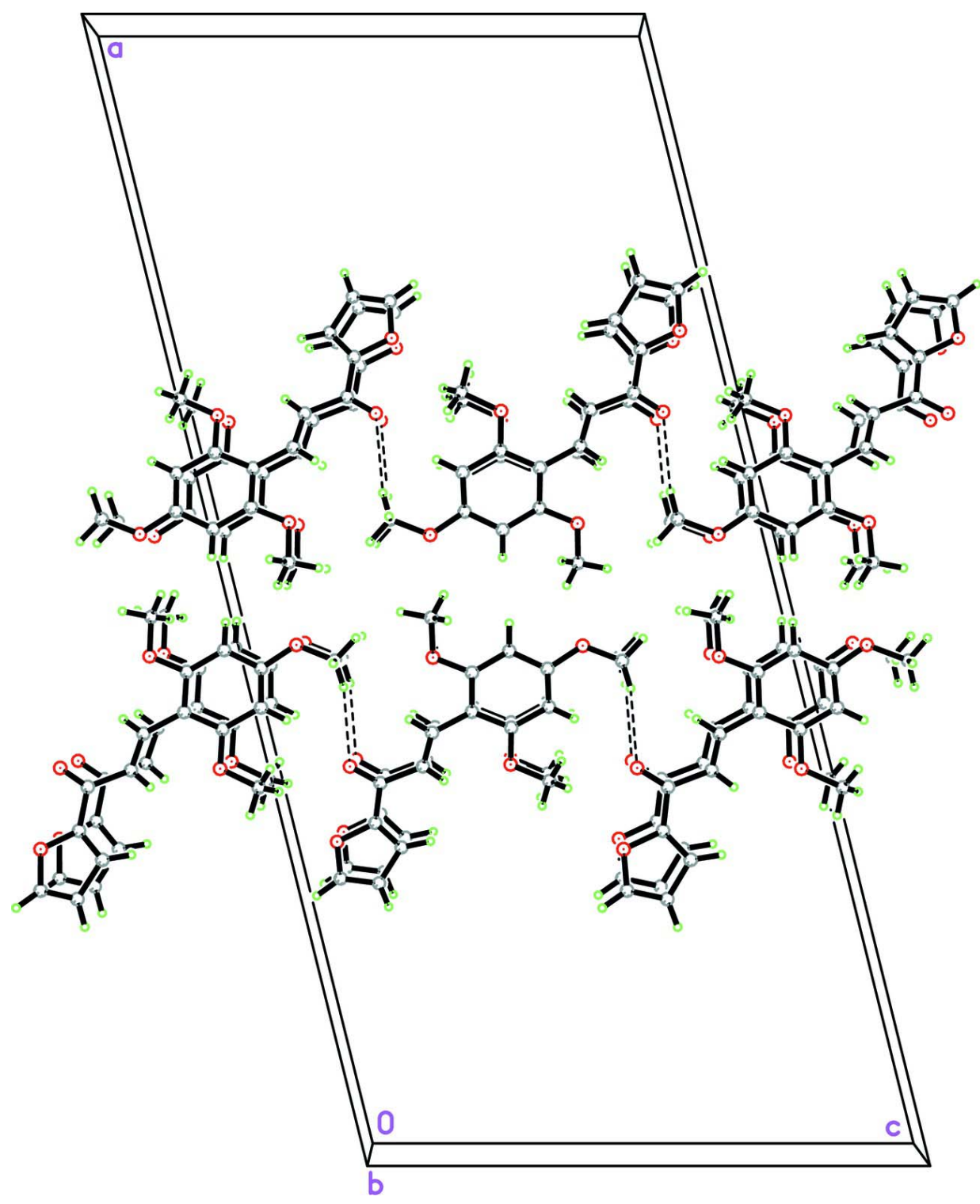

Figure 3

The crystal packing of (I) viewed along the $b$ axis, showing chains stacking along the $b$ axis. Weak $\mathrm{C}-\mathrm{H} \cdots \mathrm{O}$ interactions are shown as dashed lines.

\section{(E)-1-(2-Furyl)-3-(2,4,6-trimethoxyphenyl)prop-2-en-1-one}

\section{Crystal data}

$\mathrm{C}_{16} \mathrm{H}_{16} \mathrm{O}_{5}$

$M_{r}=288.29$

Monoclinic, $C 2 / c$

Hall symbol: $-\mathrm{C} 2 \mathrm{yc}$

$a=38.5688(5) \AA$

$b=3.93493(5) \AA$

$c=18.2638(3) \AA$

$\beta=103.901(1)^{\circ}$

$V=2690.68(6) \AA^{3}$

$Z=8$
$F(000)=1216$

$D_{\mathrm{x}}=1.423 \mathrm{Mg} \mathrm{m}^{-3}$

Melting point $=390-391 \mathrm{~K}$

Mo $K \alpha$ radiation, $\lambda=0.71073 \AA$

Cell parameters from 3941 reflections

$\theta=1.1-30.0^{\circ}$

$\mu=0.11 \mathrm{~mm}^{-1}$

$T=100 \mathrm{~K}$

Block, colorless

$0.41 \times 0.15 \times 0.09 \mathrm{~mm}$ 


\section{Data collection \\ Bruker APEXII CCD \\ diffractometer \\ Radiation source: sealed tube \\ Graphite monochromator \\ $\varphi$ and $\omega$ scans \\ Absorption correction: multi-scan \\ (SADABS; Bruker, 2005) \\ $T_{\min }=0.957, T_{\max }=0.990$}

\section{Refinement}

Refinement on $F^{2}$

Least-squares matrix: full

$R\left[F^{2}>2 \sigma\left(F^{2}\right)\right]=0.041$

$w R\left(F^{2}\right)=0.113$

$S=1.06$

3941 reflections

254 parameters

0 restraints

Primary atom site location: structure-invariant direct methods
20657 measured reflections

3941 independent reflections

3077 reflections with $I>2 \sigma(I)$

$R_{\text {int }}=0.038$

$\theta_{\text {max }}=30.0^{\circ}, \theta_{\min }=1.1^{\circ}$

$h=-54 \rightarrow 54$

$k=-5 \rightarrow 5$

$l=-25 \rightarrow 25$

Secondary atom site location: difference Fourier map

Hydrogen site location: inferred from neighbouring sites

All H-atom parameters refined

$w=1 /\left[\sigma^{2}\left(F_{\mathrm{o}}^{2}\right)+(0.0556 P)^{2}+1.4768 P\right]$

where $P=\left(F_{\mathrm{o}}^{2}+2 F_{\mathrm{c}}{ }^{2}\right) / 3$

$(\Delta / \sigma)_{\max }=0.001$

$\Delta \rho_{\max }=0.35$ e $\AA^{-3}$

$\Delta \rho_{\min }=-0.24 \mathrm{e} \AA^{-3}$

Special details

Experimental. The crystal was placed in the cold stream of an Oxford Cryosystems Cobra open-flow nitrogen cryostat (Cosier \& Glazer, 1986) operating at 120.0 (1) K.

Geometry. All esds (except the esd in the dihedral angle between two 1.s. planes) are estimated using the full covariance matrix. The cell esds are taken into account individually in the estimation of esds in distances, angles and torsion angles; correlations between esds in cell parameters are only used when they are defined by crystal symmetry. An approximate (isotropic) treatment of cell esds is used for estimating esds involving l.s. planes.

Refinement. Refinement of $\mathrm{F}^{2}$ against ALL reflections. The weighted R-factor $\mathrm{wR}$ and goodness of fit $\mathrm{S}$ are based on $\mathrm{F}^{2}$, conventional R-factors $R$ are based on $F$, with $F$ set to zero for negative $F^{2}$. The threshold expression of $F^{2}>2 \operatorname{sigma}\left(F^{2}\right)$ is used only for calculating R-factors(gt) etc. and is not relevant to the choice of reflections for refinement. R-factors based on $\mathrm{F}^{2}$ are statistically about twice as large as those based on F, and R- factors based on ALL data will be even larger.

Fractional atomic coordinates and isotropic or equivalent isotropic displacement parameters $\left(\AA^{2}\right)$

\begin{tabular}{lllll}
\hline & $x$ & $y$ & $z$ & $U_{\text {iso }} * / U_{\text {eq }}$ \\
\hline O1 & $0.15077(2)$ & $0.0988(3)$ & $0.84899(5)$ & $0.0215(2)$ \\
O2 & $0.21727(2)$ & $0.3478(2)$ & $0.90839(4)$ & $0.01663(19)$ \\
O3 & $0.05690(2)$ & $-0.2197(2)$ & $0.65467(5)$ & $0.01694(19)$ \\
O4 & $0.04708(2)$ & $-0.1724(2)$ & $0.39117(5)$ & $0.01735(19)$ \\
O5 & $0.15024(2)$ & $0.2851(2)$ & $0.56781(5)$ & $0.01639(19)$ \\
C1 & $0.25039(3)$ & $0.4913(3)$ & $0.92049(7)$ & $0.0174(2)$ \\
H1 & $0.2651(4)$ & $0.474(4)$ & $0.9706(8)$ & $0.018(4)^{*}$ \\
C2 & $0.25591(3)$ & $0.6274(3)$ & $0.85645(7)$ & $0.0188(3)$ \\
H2 & $0.2775(4)$ & $0.741(4)$ & $0.8513(9)$ & $0.027(4)^{*}$ \\
C3 & $0.22403(3)$ & $0.5659(3)$ & $0.79963(7)$ & $0.0174(2)$ \\
$\mathrm{H} 3$ & $0.2194(4)$ & $0.630(4)$ & $0.7461(9)$ & $0.021(4)^{*}$ \\
C4 & $0.20144(3)$ & $0.3929(3)$ & $0.83315(6)$ & $0.0141(2)$ \\
C5 & $0.16581(3)$ & $0.2391(3)$ & $0.80482(6)$ & $0.0150(2)$ \\
C6 & $0.15171(3)$ & $0.2521(3)$ & $0.72275(7)$ & $0.0153(2)$
\end{tabular}




$\begin{array}{lllll}\text { H6 } & 0.1649(4) & 0.381(4) & 0.6935(8) & 0.019(4)^{*} \\ \text { C7 } & 0.12181(3) & 0.0762(3) & 0.69077(6) & 0.0145(2) \\ \text { H7 } & 0.1101(4) & -0.049(4) & 0.7240(8) & 0.020(4)^{*} \\ \text { C8 } & 0.10376(3) & 0.0332(3) & 0.61194(6) & 0.0133(2) \\ \text { C9 } & 0.06997(3) & -0.1318(3) & 0.59416(6) & 0.0132(2) \\ \text { C10 } & 0.05156(3) & -0.1931(3) & 0.52043(7) & 0.0150(2) \\ \text { H10 } & 0.0282(4) & -0.310(4) & 0.5082(9) & 0.027(4)^{*} \\ \text { C11 } & 0.06682(3) & -0.0939(3) & 0.46185(6) & 0.0140(2) \\ \text { C12 } & 0.09968(3) & 0.0689(3) & 0.47557(6) & 0.0139(2) \\ \text { H12 } & 0.1100(4) & 0.127(4) & 0.4359(8) & 0.018(4)^{*} \\ \text { C13 } & 0.11766(3) & 0.1321(3) & 0.55034(6) & 0.0131(2) \\ \text { C14 } & 0.02326(3) & -0.3920(3) & 0.63942(7) & 0.0177(2) \\ \text { H14A } & 0.0040(4) & -0.255(4) & 0.6091(8) & 0.018(4)^{*} \\ \text { H14B } & 0.0253(4) & -0.612(4) & 0.6129(8) & 0.021(4)^{*} \\ \text { H14C } & 0.0183(4) & -0.431(4) & 0.6887(9) & 0.023(4)^{*} \\ \text { C15 } & 0.06015(4) & -0.0546(4) & 0.32866(7) & 0.0206(3) \\ \text { H15A } & 0.0833(4) & -0.158(4) & 0.3269(9) & 0.024(4)^{*} \\ \text { H15B } & 0.0411(5) & -0.122(4) & 0.2834(9) & 0.032(4)^{*} \\ \text { H15C } & 0.0620(4) & 0.196(4) & 0.3296(9) & 0.024(4)^{*} \\ \text { C16 } & 0.16511(3) & 0.3953(3) & 0.50694(7) & 0.0158(2) \\ \text { H16A } & 0.1703(4) & 0.200(4) & 0.4778(9) & 0.021(4)^{*} \\ \text { H16B } & 0.1490(4) & 0.555(4) & 0.4762(8) & 0.015(3)^{*} \\ \text { H16C } & 0.1875(4) & 0.511(4) & 0.5307(8) & 0.020(4)^{*} \\ & & & & \end{array}$

Atomic displacement parameters $\left(\AA^{2}\right)$

\begin{tabular}{lllllll}
\hline & $U^{11}$ & $U^{22}$ & $U^{33}$ & $U^{12}$ & $U^{13}$ & $U^{23}$ \\
\hline O1 & $0.0194(4)$ & $0.0314(5)$ & $0.0145(4)$ & $-0.0052(4)$ & $0.0057(3)$ & $0.0020(4)$ \\
O2 & $0.0151(4)$ & $0.0227(5)$ & $0.0111(4)$ & $-0.0018(3)$ & $0.0012(3)$ & $0.0000(3)$ \\
O3 & $0.0143(4)$ & $0.0231(5)$ & $0.0142(4)$ & $-0.0047(3)$ & $0.0049(3)$ & $0.0000(3)$ \\
O4 & $0.0162(4)$ & $0.0239(5)$ & $0.0111(4)$ & $-0.0038(3)$ & $0.0015(3)$ & $-0.0018(3)$ \\
O5 & $0.0130(4)$ & $0.0242(5)$ & $0.0119(4)$ & $-0.0051(3)$ & $0.0029(3)$ & $0.0009(3)$ \\
C1 & $0.0152(5)$ & $0.0204(6)$ & $0.0157(6)$ & $-0.0013(4)$ & $0.0017(4)$ & $-0.0018(5)$ \\
C2 & $0.0174(6)$ & $0.0211(6)$ & $0.0182(6)$ & $-0.0039(5)$ & $0.0049(5)$ & $-0.0022(5)$ \\
C3 & $0.0193(6)$ & $0.0193(6)$ & $0.0133(5)$ & $-0.0018(4)$ & $0.0037(4)$ & $-0.0014(5)$ \\
C4 & $0.0152(5)$ & $0.0171(6)$ & $0.0094(5)$ & $0.0016(4)$ & $0.0016(4)$ & $-0.0009(4)$ \\
C5 & $0.0149(5)$ & $0.0171(6)$ & $0.0131(5)$ & $0.0011(4)$ & $0.0038(4)$ & $-0.0007(4)$ \\
C6 & $0.0155(5)$ & $0.0172(6)$ & $0.0131(5)$ & $-0.0002(4)$ & $0.0033(4)$ & $0.0004(4)$ \\
C7 & $0.0146(5)$ & $0.0169(6)$ & $0.0122(5)$ & $0.0018(4)$ & $0.0038(4)$ & $-0.0003(4)$ \\
C8 & $0.0129(5)$ & $0.0147(5)$ & $0.0124(5)$ & $0.0009(4)$ & $0.0032(4)$ & $-0.0001(4)$ \\
C9 & $0.0135(5)$ & $0.0139(5)$ & $0.0129(5)$ & $0.0006(4)$ & $0.0048(4)$ & $0.0008(4)$ \\
C10 & $0.0125(5)$ & $0.0170(6)$ & $0.0150(5)$ & $-0.0007(4)$ & $0.0027(4)$ & $-0.0006(4)$ \\
C11 & $0.0132(5)$ & $0.0153(6)$ & $0.0121(5)$ & $0.0011(4)$ & $0.0003(4)$ & $-0.0012(4)$ \\
C12 & $0.0133(5)$ & $0.0163(6)$ & $0.0121(5)$ & $0.0014(4)$ & $0.0031(4)$ & $0.0006(4)$ \\
C13 & $0.0106(5)$ & $0.0133(5)$ & $0.0152(5)$ & $-0.0001(4)$ & $0.0028(4)$ & $0.0002(4)$ \\
C14 & $0.0142(5)$ & $0.0197(6)$ & $0.0199(6)$ & $-0.0029(4)$ & $0.0058(5)$ & $0.0018(5)$ \\
C15 & $0.0232(6)$ & $0.0265(7)$ & $0.0117(5)$ & $-0.0043(5)$ & $0.0036(5)$ & $0.0000(5)$ \\
C16 & $0.0143(5)$ & $0.0205(6)$ & $0.0133(5)$ & $-0.0023(4)$ & $0.0043(4)$ & $0.0024(5)$ \\
& & & & & &
\end{tabular}


Geometric parameters $\left(\AA,{ }^{\circ}\right)$

\begin{tabular}{|c|c|c|c|}
\hline $\mathrm{O} 1-\mathrm{C} 5$ & $1.2315(14)$ & $\mathrm{C} 7-\mathrm{C} 8$ & 1.4507 (16) \\
\hline $\mathrm{O} 2-\mathrm{C} 1$ & $1.3652(14)$ & $\mathrm{C} 7-\mathrm{H} 7$ & $0.974(15)$ \\
\hline $\mathrm{O} 2-\mathrm{C} 4$ & $1.3745(13)$ & $\mathrm{C} 8-\mathrm{C} 13$ & $1.4123(15)$ \\
\hline $\mathrm{O} 3-\mathrm{C} 9$ & $1.3648(13)$ & $\mathrm{C} 8-\mathrm{C} 9$ & $1.4220(15)$ \\
\hline $\mathrm{O} 3-\mathrm{C} 14$ & $1.4307(14)$ & $\mathrm{C} 9-\mathrm{C} 10$ & $1.3844(16)$ \\
\hline $\mathrm{O} 4-\mathrm{C} 11$ & $1.3673(14)$ & $\mathrm{C} 10-\mathrm{C} 11$ & $1.3949(16)$ \\
\hline $\mathrm{O} 4-\mathrm{C} 15$ & $1.4317(15)$ & $\mathrm{C} 10-\mathrm{H} 10$ & $0.988(16)$ \\
\hline $\mathrm{O} 5-\mathrm{C} 13$ & $1.3602(13)$ & $\mathrm{C} 11-\mathrm{C} 12$ & $1.3883(16)$ \\
\hline $\mathrm{O} 5-\mathrm{C} 16$ & $1.4352(14)$ & $\mathrm{C} 12-\mathrm{C} 13$ & $1.3974(16)$ \\
\hline $\mathrm{C} 1-\mathrm{C} 2$ & $1.3492(17)$ & $\mathrm{C} 12-\mathrm{H} 12$ & $0.936(15)$ \\
\hline $\mathrm{C} 1-\mathrm{H} 1$ & $0.956(15)$ & $\mathrm{C} 14-\mathrm{H} 14 \mathrm{~A}$ & $0.976(15)$ \\
\hline $\mathrm{C} 2-\mathrm{C} 3$ & $1.4264(17)$ & C14-H14B & $1.003(16)$ \\
\hline $\mathrm{C} 2-\mathrm{H} 2$ & $0.970(16)$ & $\mathrm{C} 14-\mathrm{H} 14 \mathrm{C}$ & $0.977(16)$ \\
\hline $\mathrm{C} 3-\mathrm{C} 4$ & $1.3617(17)$ & C15-H15A & $0.989(16)$ \\
\hline $\mathrm{C} 3-\mathrm{H} 3$ & $0.984(15)$ & C15-H15B & $1.001(17)$ \\
\hline $\mathrm{C} 4-\mathrm{C} 5$ & $1.4766(16)$ & $\mathrm{C} 15-\mathrm{H} 15 \mathrm{C}$ & $0.990(17)$ \\
\hline $\mathrm{C} 5-\mathrm{C} 6$ & $1.4675(16)$ & $\mathrm{C} 16-\mathrm{H} 16 \mathrm{~A}$ & $0.982(16)$ \\
\hline $\mathrm{C} 6-\mathrm{C} 7$ & $1.3512(16)$ & C16-H16B & $0.963(15)$ \\
\hline C6-H6 & $0.967(15)$ & $\mathrm{C} 16-\mathrm{H} 16 \mathrm{C}$ & $0.982(16)$ \\
\hline $\mathrm{C} 1-\mathrm{O} 2-\mathrm{C} 4$ & $106.39(9)$ & $\mathrm{C} 9-\mathrm{C} 10-\mathrm{C} 11$ & $119.01(10)$ \\
\hline $\mathrm{C} 9-\mathrm{O} 3-\mathrm{C} 14$ & $117.18(9)$ & $\mathrm{C} 9-\mathrm{C} 10-\mathrm{H} 10$ & $121.8(9)$ \\
\hline $\mathrm{C} 11-\mathrm{O} 4-\mathrm{C} 15$ & $117.18(9)$ & $\mathrm{C} 11-\mathrm{C} 10-\mathrm{H} 10$ & $119.2(9)$ \\
\hline $\mathrm{C} 13-\mathrm{O} 5-\mathrm{C} 16$ & $118.08(9)$ & $\mathrm{O} 4-\mathrm{C} 11-\mathrm{C} 12$ & $123.50(10)$ \\
\hline $\mathrm{C} 2-\mathrm{C} 1-\mathrm{O} 2$ & $111.12(10)$ & $\mathrm{O} 4-\mathrm{C} 11-\mathrm{C} 10$ & $114.76(10)$ \\
\hline $\mathrm{C} 2-\mathrm{C} 1-\mathrm{H} 1$ & $132.6(9)$ & $\mathrm{C} 12-\mathrm{C} 11-\mathrm{C} 10$ & $121.74(10)$ \\
\hline $\mathrm{O} 2-\mathrm{C} 1-\mathrm{H} 1$ & $116.2(9)$ & $\mathrm{C} 11-\mathrm{C} 12-\mathrm{C} 13$ & $118.39(10)$ \\
\hline $\mathrm{C} 1-\mathrm{C} 2-\mathrm{C} 3$ & $105.98(11)$ & $\mathrm{C} 11-\mathrm{C} 12-\mathrm{H} 12$ & $120.9(9)$ \\
\hline $\mathrm{C} 1-\mathrm{C} 2-\mathrm{H} 2$ & $125.8(10)$ & $\mathrm{C} 13-\mathrm{C} 12-\mathrm{H} 12$ & $120.7(9)$ \\
\hline $\mathrm{C} 3-\mathrm{C} 2-\mathrm{H} 2$ & $128.2(10)$ & $\mathrm{O} 5-\mathrm{C} 13-\mathrm{C} 12$ & $121.43(10)$ \\
\hline $\mathrm{C} 4-\mathrm{C} 3-\mathrm{C} 2$ & $106.90(11)$ & $\mathrm{O} 5-\mathrm{C} 13-\mathrm{C} 8$ & $116.18(10)$ \\
\hline $\mathrm{C} 4-\mathrm{C} 3-\mathrm{H} 3$ & $126.4(9)$ & $\mathrm{C} 12-\mathrm{C} 13-\mathrm{C} 8$ & $122.37(10)$ \\
\hline $\mathrm{C} 2-\mathrm{C} 3-\mathrm{H} 3$ & 126.7 (9) & $\mathrm{O} 3-\mathrm{C} 14-\mathrm{H} 14 \mathrm{~A}$ & $112.3(9)$ \\
\hline $\mathrm{C} 3-\mathrm{C} 4-\mathrm{O} 2$ & $109.60(10)$ & $\mathrm{O} 3-\mathrm{C} 14-\mathrm{H} 14 \mathrm{~B}$ & $109.3(8)$ \\
\hline $\mathrm{C} 3-\mathrm{C} 4-\mathrm{C} 5$ & $133.59(11)$ & $\mathrm{H} 14 \mathrm{~A}-\mathrm{C} 14-\mathrm{H} 14 \mathrm{~B}$ & $109.8(12)$ \\
\hline $\mathrm{O} 2-\mathrm{C} 4-\mathrm{C} 5$ & $116.74(10)$ & $\mathrm{O} 3-\mathrm{C} 14-\mathrm{H} 14 \mathrm{C}$ & $105.3(9)$ \\
\hline $\mathrm{O} 1-\mathrm{C} 5-\mathrm{C} 6$ & $124.57(11)$ & $\mathrm{H} 14 \mathrm{~A}-\mathrm{C} 14-\mathrm{H} 14 \mathrm{C}$ & $108.5(12)$ \\
\hline $\mathrm{O} 1-\mathrm{C} 5-\mathrm{C} 4$ & $119.95(10)$ & $\mathrm{H} 14 \mathrm{~B}-\mathrm{C} 14-\mathrm{H} 14 \mathrm{C}$ & $111.5(13)$ \\
\hline $\mathrm{C} 6-\mathrm{C} 5-\mathrm{C} 4$ & $115.40(10)$ & $\mathrm{O} 4-\mathrm{C} 15-\mathrm{H} 15 \mathrm{~A}$ & $112.7(9)$ \\
\hline $\mathrm{C} 7-\mathrm{C} 6-\mathrm{C} 5$ & $119.48(11)$ & $\mathrm{O} 4-\mathrm{C} 15-\mathrm{H} 15 \mathrm{~B}$ & $104.0(10)$ \\
\hline $\mathrm{C} 7-\mathrm{C} 6-\mathrm{H} 6$ & $122.6(9)$ & $\mathrm{H} 15 \mathrm{~A}-\mathrm{C} 15-\mathrm{H} 15 \mathrm{~B}$ & $110.8(13)$ \\
\hline $\mathrm{C} 5-\mathrm{C} 6-\mathrm{H} 6$ & $117.9(9)$ & $\mathrm{O} 4-\mathrm{C} 15-\mathrm{H} 15 \mathrm{C}$ & $110.3(9)$ \\
\hline $\mathrm{C} 6-\mathrm{C} 7-\mathrm{C} 8$ & $130.17(11)$ & $\mathrm{H} 15 \mathrm{~A}-\mathrm{C} 15-\mathrm{H} 15 \mathrm{C}$ & $110.6(13)$ \\
\hline $\mathrm{C} 6-\mathrm{C} 7-\mathrm{H} 7$ & $117.8(9)$ & $\mathrm{H} 15 \mathrm{~B}-\mathrm{C} 15-\mathrm{H} 15 \mathrm{C}$ & $108.2(14)$ \\
\hline $\mathrm{C} 8-\mathrm{C} 7-\mathrm{H} 7$ & $112.0(9)$ & $\mathrm{O} 5-\mathrm{C} 16-\mathrm{H} 16 \mathrm{~A}$ & $110.8(9)$ \\
\hline $\mathrm{C} 13-\mathrm{C} 8-\mathrm{C} 9$ & $116.51(10)$ & $\mathrm{O} 5-\mathrm{C} 16-\mathrm{H} 16 \mathrm{~B}$ & $109.1(8)$ \\
\hline
\end{tabular}




$\begin{array}{llll}\mathrm{C} 13-\mathrm{C} 8-\mathrm{C} 7 & 125.09(10) & \mathrm{H} 16 \mathrm{~A}-\mathrm{C} 16-\mathrm{H} 16 \mathrm{~B} & 112.5(12) \\ \mathrm{C} 9-\mathrm{C} 8-\mathrm{C} 7 & 118.36(10) & \mathrm{O} 5-\mathrm{C} 16-\mathrm{H} 16 \mathrm{C} & 105.8(8) \\ \mathrm{O} 3-\mathrm{C} 9-\mathrm{C} 10 & 122.72(10) & \mathrm{H} 16 \mathrm{~A}-\mathrm{C} 16-\mathrm{H} 16 \mathrm{C} & 109.3(12) \\ \mathrm{O} 3-\mathrm{C} 9-\mathrm{C} 8 & 115.31(10) & \mathrm{H} 16 \mathrm{~B}-\mathrm{C} 16-\mathrm{H} 16 \mathrm{C} & 109.2(13) \\ \mathrm{C} 10-\mathrm{C} 9-\mathrm{C} 8 & 121.96(10) & & \\ & & & 3.17(16) \\ \mathrm{C} 4-\mathrm{O} 2-\mathrm{C} 1-\mathrm{C} 2 & 0.63(14) & \mathrm{C} 7-\mathrm{C} 8-\mathrm{C} 9-\mathrm{O} 3 & 0.06(17) \\ \mathrm{O} 2-\mathrm{C} 1-\mathrm{C} 2-\mathrm{C} 3 & -0.01(15) & \mathrm{C} 13-\mathrm{C} 8-\mathrm{C} 9-\mathrm{C} 10 & -177.77(11) \\ \mathrm{C} 1-\mathrm{C} 2-\mathrm{C} 3-\mathrm{C} 4 & -0.63(14) & \mathrm{C} 7-\mathrm{C} 8-\mathrm{C} 9-\mathrm{C} 10 & 179.79(11) \\ \mathrm{C} 2-\mathrm{C} 3-\mathrm{C} 4-\mathrm{O} 2 & 1.03(14) & \mathrm{O} 3-\mathrm{C} 9-\mathrm{C} 10-\mathrm{C} 11 & 0.80(18) \\ \mathrm{C} 2-\mathrm{C} 3-\mathrm{C} 4-\mathrm{C} 5 & -175.73(13) & \mathrm{C} 8-\mathrm{C} 9-\mathrm{C} 10-\mathrm{C} 11 & -5.04(17) \\ \mathrm{C} 1-\mathrm{O} 2-\mathrm{C} 4-\mathrm{C} 3 & -1.03(13) & \mathrm{C} 15-\mathrm{O} 4-\mathrm{C} 11-\mathrm{C} 12 & 175.53(11) \\ \mathrm{C} 1-\mathrm{O} 2-\mathrm{C} 4-\mathrm{C} 5 & 176.35(10) & \mathrm{C} 15-\mathrm{O} 4-\mathrm{C} 11-\mathrm{C} 10 & -0.95(10) \\ \mathrm{C} 3-\mathrm{C} 4-\mathrm{C} 5-\mathrm{O} 1 & -178.75(13) & \mathrm{C} 9-\mathrm{C} 10-\mathrm{C} 11-\mathrm{O} 4 & -179.17(11) \\ \mathrm{O} 2-\mathrm{C} 4-\mathrm{C} 5-\mathrm{O} 1 & 4.67(17) & \mathrm{C} 9-\mathrm{C} 10-\mathrm{C} 11-\mathrm{C} 12 & 0.22(18) \\ \mathrm{C} 3-\mathrm{C} 4-\mathrm{C} 5-\mathrm{C} 6 & 4.4(2) & \mathrm{O} 4-\mathrm{C} 11-\mathrm{C} 12-\mathrm{C} 13 & 2.85(16) \\ \mathrm{O} 2-\mathrm{C} 4-\mathrm{C} 5-\mathrm{C} 6 & -172.18(10) & \mathrm{C} 10-\mathrm{C} 11-\mathrm{C} 12-\mathrm{C} 13 & -178.91(10) \\ \mathrm{O} 1-\mathrm{C} 5-\mathrm{C} 6-\mathrm{C} 7 & -6.08(19) & \mathrm{C} 16-\mathrm{O} 5-\mathrm{C} 13-\mathrm{C} 12 & 178.81(10) \\ \mathrm{C} 4-\mathrm{C} 5-\mathrm{C} 6-\mathrm{C} 7 & 170.60(11) & \mathrm{C} 16-\mathrm{O}-\mathrm{C} 13-\mathrm{C} 8 & 0.69(18) \\ \mathrm{C} 5-\mathrm{C} 6-\mathrm{C} 7-\mathrm{C} 8 & -176.44(12) & \mathrm{C} 11-\mathrm{C} 12-\mathrm{C} 13-\mathrm{O} 5 & -179.03(10) \\ \mathrm{C} 6-\mathrm{C} 7-\mathrm{C} 8-\mathrm{C} 13 & 10.2(2) & \mathrm{C} 11-\mathrm{C} 12-\mathrm{C} 13-\mathrm{C} 8 & -1.37(17) \\ \mathrm{C} 6-\mathrm{C} 7-\mathrm{C} 8-\mathrm{C} 9 & -172.18(12) & \mathrm{C} 9-\mathrm{C} 8-\mathrm{C} 13-\mathrm{O} 5 & -0.82(17) \\ \mathrm{C} 14-\mathrm{O} 3-\mathrm{C} 9-\mathrm{C} 10 & 2.07(17) & \mathrm{C} 7-\mathrm{C} 8-\mathrm{C} 13-\mathrm{O} 5 & 176.84(11) \\ \mathrm{C} 14-\mathrm{O} 3-\mathrm{C} 9-\mathrm{C} 8 & -178.87(10) & \mathrm{C} 9-\mathrm{C} 8-\mathrm{C} 13-\mathrm{C} 12 & \\ \mathrm{C} 13-\mathrm{C} 8-\mathrm{C} 9-\mathrm{O} 3 & -179.00(10) & \mathrm{C} 7-\mathrm{C} 8-\mathrm{C} 13-\mathrm{C} 12 & \\ & & & \end{array}$

Hydrogen-bond geometry $\left(\AA,{ }^{\circ}\right)$

\begin{tabular}{lllll}
\hline$D-\mathrm{H} \cdots A$ & $D-\mathrm{H}$ & $\mathrm{H} \cdots A$ & $D \cdots A$ & $D-\mathrm{H} \cdots A$ \\
\hline $\mathrm{C} 1-\mathrm{H} 1 \cdots \mathrm{O} 2^{\mathrm{i}}$ & $0.956(15)$ & $2.496(15)$ & $3.3512(14)$ & $148.9(13)$ \\
$\mathrm{C} 6-\mathrm{H} 6 \cdots \mathrm{O} 5$ & $0.965(15)$ & $2.260(14)$ & $2.8197(15)$ & $116.0(11)$ \\
$\mathrm{C} 14-\mathrm{H} 14 A \cdots \mathrm{O} 44^{\mathrm{ii}}$ & $0.975(15)$ & $2.589(16)$ & $3.4462(14)$ & $146.7(11)$ \\
$\mathrm{C} 15-\mathrm{H} 15 A \cdots \mathrm{O} 1^{\mathrm{iii}}$ & $0.989(16)$ & $2.546(16)$ & $3.4293(18)$ & $148.6(12)$ \\
$\mathrm{C} 16-\mathrm{H} 16 A \cdots \mathrm{O} 1^{\mathrm{iii}}$ & $0.982(16)$ & $2.575(16)$ & $3.4120(16)$ & $143.0(12)$
\end{tabular}

Symmetry codes: (i) $-x+1 / 2,-y+1 / 2,-z+2$; (ii) $-x,-y,-z+1$; (iii) $x,-y, z-1 / 2$. 